\title{
Explaining the relation between pathological gambling and depression: Rumination as an underlying common cause
}

\author{
KRISTIAN KRAUSE ${ }^{1}$, ANJA BISCHOF ${ }^{2}$, SILVIA LEWIN $^{3}$, DIANA GUERTLER ${ }^{1,4}$, HANS-JÜRGEN RUMPF ${ }^{2}$, \\ ULRICH JOHN ${ }^{1,4}$ and CHRISTIAN MEYER ${ }^{1,4}$ \\ ${ }^{1}$ Institute of Social Medicine and Prevention, University Medicine Greifswald, Greifswald, Germany \\ ${ }^{2}$ Department of Psychiatry and Psychotherapy, Research Group S:TEP, University of Lübeck, Lübeck, Germany \\ ${ }^{3}$ Klinik Niederrhein, Psychological Service, Bad Neuenahr-Ahrweiler, Germany \\ ${ }^{4}$ DZHK (German Centre for Cardiovascular Research), Partner Site Greifswald, Greifswald, Germany
}

(Received: December 22, 2017; revised manuscript received: April 6, 2018; accepted: April 7, 2018)

\begin{abstract}
Background and aims: Symptoms of pathological gambling (SPG) and depression often co-occur. The nature of this relationship remains unclear. Rumination, which is well known to be associated with depression, might act as a common underlying factor explaining the frequent co-occurrence of both conditions. The aim of this study is to analyze associations between the rumination subfactors brooding and reflection and SPG. Methods: Participants aged 14-64 years were recruited within an epidemiological study on pathological gambling in Germany. Cross-sectional data of 506 ( $80.4 \%$ male) individuals with a history of gambling problems were analyzed. The assessment included a standardized clinical interview. To examine the effects of rumination across different levels of problem gambling severity, sequential quantile regression was used to analyze the association between the rumination subfactors and SPG. Results: Brooding ( $p=.005$ ) was positively associated with the severity of problem gambling after adjusting for reflection, depressive symptoms, and sociodemographic variables. Along the distribution of problem gambling severity, findings hold for all but the lowest severity level. Reflection $(p=.347)$ was not associated with the severity of problem gambling at the median. Along the distribution of problem gambling severity, there was an inverse association at only one quantile. Discussion and conclusions: Brooding might be important in the development and maintenance of problem gambling. With its relations to depression and problem gambling, it might be crucial when it comes to explaining the high comorbidity rates between SPG and depression. The role of reflection in SPG remains inconclusive.
\end{abstract}

Keywords: rumination, pathological gambling, depression, brooding, reflection

\section{INTRODUCTION}

It has been shown that symptoms of pathological gambling (SPG) and symptoms of depression often co-occur (Kessler et al., 2008; Petry, Stinson, \& Grant, 2005; Rizeanu, 2013). However, the nature of this relationship remains unclear. One possible explanation for the high comorbidity is that SPG and symptoms of depression pose risk-factors for one another. As for the question of which one is preceding which, there have been conflicting results (Afifi, Nicholson, Martins, \& Sareen, 2016; Kessler et al., 2008). Analyzing data from a longitudinal study on the relationship between gambling problems and depression in a sample of young adults aged 18-20, Chinneck, Mackinnon, and Stewart (2016) concluded that the frequent co-occurrence of gambling problems and depressive disorders is not due to one of them being a risk-factor for the other, but due to an underlying factor that both disorders have in common. People's response styles (Nolen-Hoeksema, 1991) might be such a factor.

According to Response Style Theory (Nolen-Hoeksema, 1991), the way people react to depressed mood states is an important factor when it comes to the severity and duration of such states. While engaging in distracting activities helps to reduce and to shorten depressive symptoms, rumination, that is to think about possible causes and implications of one's mood, leads to an amplification and continuation of such symptoms. Treynor, Gonzalez, and Nolen-Hoeksema (2003) later refined the concept of rumination. They found that rumination consists of two distinct factors, which differentially relate to depressive symptoms. They named these factors brooding and reflection. Brooding thereby reflects " $a$ passive comparison of one's current situation with some unachieved standard" (Treynor et al., 2003, p. 256). Reflection on the other hand reflects "a purposeful turning inward to engage in cognitive problem solving to alleviate one's depressive symptoms" (Treynor et al., 2003, p. 256).

\footnotetext{
* Corresponding author: Kristian Krause, Dipl.-Psych.; Institute of Social Medicine and Prevention, University Medicine Greifswald, Walther-Rathenau-Str. 48, Greifswald D-17475, Germany; Phone: +49 3834867 722; Fax: +49 3834867 701; E-mail: kristian. krause@uni-greifswald.de
}

This is an open-access article distributed under the terms of the Creative Commons Attribution-NonCommercial 4.0 International License, which permits unrestricted use, distribution, and reproduction in any medium for non-commercial purposes, provided the original author and source are credited, a link to the CC License is provided, and changes - if any - are indicated. 
While both factors correlate with current depression, only brooding correlates with depression over time, which led Treynor et al. (2003) to the assumption that reflection might be adaptive in reducing depressive symptoms over time by causing effective problem solving.

It has been shown that rumination is not only related to depression but to several maladaptive behaviors as well, including binge drinking, alcohol abuse, binge eating, and self-harm (Nolen-Hoeksema, Wisco, \& Lyubomirsky, 2008). There is evidence that rumination, especially its subfactor brooding, might be related to gambling as well. Using the Problem-Focused Styles of Coping Inventory (PF-SOC; Heppner, Cook, Wright, \& Johnson, 1995), Getty, Watson, and Frisch (2000) assessed the coping styles of pathological gamblers of the Gamblers Anonymous (GA) and compared them with the coping styles of non-pathological gambling matched controls. They found that pathological gamblers reported higher levels of reactive (ruminative) coping and lower levels of reflective coping. Most importantly, the differences in reactive coping could not be explained by depressive symptoms. While the PF-SOC does not directly assess rumination in the sense of the Response Style Theory (Nolen-Hoeksema, 1991), the results hint at the importance of rumination, especially brooding, in SPG, as the reactive and reflective coping styles measured with the PF-SOC show resemblance with the brooding and reflection subfactors of rumination (Getty et al., 2000).

Gambling has long been thought of as a means to alleviate depressive symptoms (McCormick, Russo, Ramirez, \& Taber, 1984), a notion supported by several studies (e.g., Dickerson, Baron, Hong, \& Cottrell, 1996; Griffiths, 1995). The results by Getty et al. (2000) suggest that there is a direct link between gambling and rumination, especially its subfactor brooding, as well. The aim of this study is to analyze the relationship between rumination and gambling problems among subjects representing a broad range of severity levels of problem gambling. In light of the aforementioned results, we expect that ruminative brooding (RB) but not ruminative reflection (RR) is related to SPG, independent from depression.

\section{METHODS}

\section{Sample}

Data were obtained from an epidemiological study on pathological gambling [Pathological Gambling and Epidemiology (PAGE); Meyer et al., 2015] in Germany. Proactive and reactive recruitment strategies were used to reach a sufficient number of participants representing different levels of gambling problems. Participants were drawn from the general population and from populations with a high risk of gambling problems, i.e., gambling location visitors, people undergoing treatment for pathological gambling, or volunteers responding to media announcements (for further details, see Meyer et al., 2015). Participants from the general population, gambling location visitors, and people undergoing treatment for pathological gambling were proactively recruited as study personnel actively reached out to them in their respective settings. The remaining participants, e.g., those responding to media announcements, were classified as being reactively recruited. Data were acquired in a two-step procedure. First, gambling behavior and criteria for pathological gambling were assessed in a diagnostic interview mostly administered through telephone. People reporting gambling problems (fulfilling one or more DSM-IV criteria for pathological gambling) were then invited to participate in an in-depth clinical interview conducted face-to-face. Additional data were collected using paper-pencil questionnaires. Gambling location visitors were offered an incentive of up to $20 €$ for their participation in the initial diagnostic interview. For participation in the in-depth clinical interview, all participants were offered an incentive of $100 €$ (c.f. Meyer et al., 2015).

\section{Measures}

Symptoms of pathological gambling. SPG were assessed using the gambling section of the World Mental Health Composite International Diagnostic Interview (CIDI) version 3.0 (World Health Organization, 2009). In general, the CIDI gambling section was administered in a computer-assisted telephone interview. For participants who were recruited in inpatient facilities, it was included in the clinical interview, which was held as a computer-assisted personal interview at the inpatient facility. Problem gambling severity was operationalized by the number of DSM-IV criteria for pathological gambling. Participants were classified as at-risk (1 to 2 DSM-IV criteria), problem (3 to 4 DSM-IV criteria), or pathological gamblers (5 or more DSM-IV criteria).

Ruminative brooding and reflection. Rumination including its subfactors brooding and reflection was assessed using the German version of the 10-item Rumination Response Scale (Treynor et al., 2003; German version RSQ10-D, Huffziger \& Kühner, 2012). Each subscale is assessed with five items, which are answered on a Likert scale ranging from 1 - almost never to 4 -almost ever. Both subscale scores thus range from 4 to 20. Huffziger and Kühner (2012) reported acceptable internal consistencies (Cronbach's $\alpha$ ) of $\alpha=.60-.75$ for the brooding subscale and $\alpha=.56-.75$ for the reflection subscale. For our sample, we found acceptable to good internal consistencies of $\alpha=.80$ for the brooding subscale and $\alpha=.73$ for the reflection subscale. The RSQ-10D was administered as a paper-pencil questionnaire.

Depressive symptoms. Depressive symptoms were assessed using the depression section of the Munich Composite International Diagnostic Interview (M-CIDI; Wittchen et al., 1995), a German language version of the WHO CIDI. The M-CIDI depression section was administered face-to-face. To determine severity levels of depression, a count of DSM-IV major depression symptoms (range: 0-9) was calculated.

\section{Statistical analysis}

All calculations were carried out using the Stata 14 statistical software package (StataCorp, 2015). Means 
were calculated for RB, RR, and the number of depressive symptoms for participants classified as at-risk, problem, and pathological gamblers. Mean differences between these groups were tested using one-way analyses of variance with subsequent Bonferroni adjusted post-hoc pairwise comparisons. To analyze the relationship between SPG (dependent variable) and both rumination subfactors (independent variables), we ran quantile regressions (QRs) using Stata's qreg procedure. QR allows to model the conditional median or any other quantile, whereas ordinary least squares (OLS) regression provides estimates of conditional means (Koenker, 2005). The estimation of QR coefficients is based on minimizing the absolute residuals rather than the sum of the squared residuals as in ordinary regression. QR was preferred over OLS regression because of the multimodal and leftskewed distribution of SPG as the dependent measure in our sample. This is in contrast to the right-skewed distribution in the general population and a consequence of the sampling design including additional recruitment channels with an increased prevalence of individuals with severe gambling problems. While normality is a critical assumption in OLS, QR allows to adequately analyze such skewed and non-normal data. Another advantage of QR is that it allows us to explore associations along the whole distribution of the dependent variable by running a series of separate QRs for different quantiles (Cameron \& Trivedi, 2009).

In this study, we first analyzed the association of SPG with the rumination subfactors and each of the other covariates using univariate median regression analysis. Then, a multivariate median regression was computed with depression severity (number of DSM-IV symptoms), sociodemographics (age, sex, education level, marital status, and migration background), and recruitment strategy (proactive vs. reactive) included as covariates besides both rumination subfactors. Finally, the multivariate QR was repeated with quantiles representing the number of SPG in our sample. To determine those quantiles, we calculated the cumulative percentages of participants reporting the respective number of symptoms. This quantile selection allows us to specifically analyze the relevance of the independent variables at any severity level represented by the dependent variable. For each quantile, a separate multivariate QR was conducted. Finally, the estimates of the QR coefficients for ruminative brooding and reflection from those analyses were plotted with $95 \%$ confidence intervals to visualize the variability across different levels of problem gambling severity.

\section{Ethics}

The study was approved by the Institutional Review Board of the University of Greifswald. All participants were informed that their participation was voluntary and that consent could be withdrawn at any time. Oral consent to participate and to store contact data was obtained during the telephone interviews. An informed consent form was signed by all participants during the clinical interview and by parents of minors.

\section{RESULTS}

\section{Description of the sample}

Participant flow and sociodemographic characteristics. Our analysis is based on a data set consisting of data from 506 individuals. Table 1 provides an overview on the sociodemographic characteristics of the sample and the number of participants recruited via proactive and reactive recruitment strategies. Figure 1 shows the participant flow. A total number of $n=15,775$ participants completed the initial diagnostic interview. Among them, 95.23\% had been recruited by the general population sample contacted through landline or mobile phone, $1.92 \%$ from gambling locations, $1.77 \%$ from treatment facilities for pathological gambling, and $1.07 \%$ through media announcements. Of the $n=15,775$ individuals, $1,796(11.39 \%)$ reported at least one SPG and were therefore eligible for the in-depth clinical interview. Of those eligible, $1,188(66.15 \%)$ individuals consented to participate in the in-depth clinical interview. For logistic reasons, the interviews were restricted to $594(50 \%)$ of those individuals. After exclusion of participants with incomplete data, $506(85.2 \%)$ participants remained in the final analysis sample. For further details on the selection process, see Meyer et al. (2015).

Symptoms of pathological gambling and depression. Participants reported a mean of $6.74(S D=2.96) \mathrm{SPG}$. The majority of the sample $(n=375,74.11 \%)$ has to be classified as pathological gamblers (5-10 SPG), followed by at-risk (1-2 SPG; $n=70,13.83 \%$ ) and problem gamblers (3-4 SPG; $n=61,12.06 \%$; c.f. Table 2).

Depressive symptoms were reported by 352 (69.57\%) participants. Percentage of individuals with at least one depressive symptom is highest among pathological gamblers $(73.33 \%, n=275)$, followed by problem $(59.02 \%$, $n=36)$ and at-risk gamblers $(58.57 \%, n=41)$. Overall,

Table 1. Sociodemographic characteristics of the sample and number of participants recruited via proactive and reactive recruitment strategies

\begin{tabular}{llrr}
\hline & & $n$ & $\%$ \\
\hline Sex & Female & 99 & 19.57 \\
& Male & 407 & 80.43 \\
Age $[M(S D)]$ & $41.18(12.17)$ & & \\
Marital status & Married/same-sex union & 144 & 28.46 \\
& Single & 244 & 48.22 \\
& Separated/divorced/ & 118 & 23.32 \\
& widowed & & \\
Employment status & Employed & 316 & 62.45 \\
& Unemployed & 190 & 37.55 \\
Education & $>10$ years & 186 & 36.76 \\
& 10 years & 169 & 33.40 \\
& $<10$ years & 142 & 28.06 \\
& Still at school & 5 & 0.99 \\
& Others & 4 & 0.79 \\
Migration & No & 372 & 73.52 \\
background & Yes & 134 & 26.48 \\
Recruitment & Proactive & 236 & 46.64 \\
strategy & Reactive & 270 & 53.36 \\
\hline
\end{tabular}




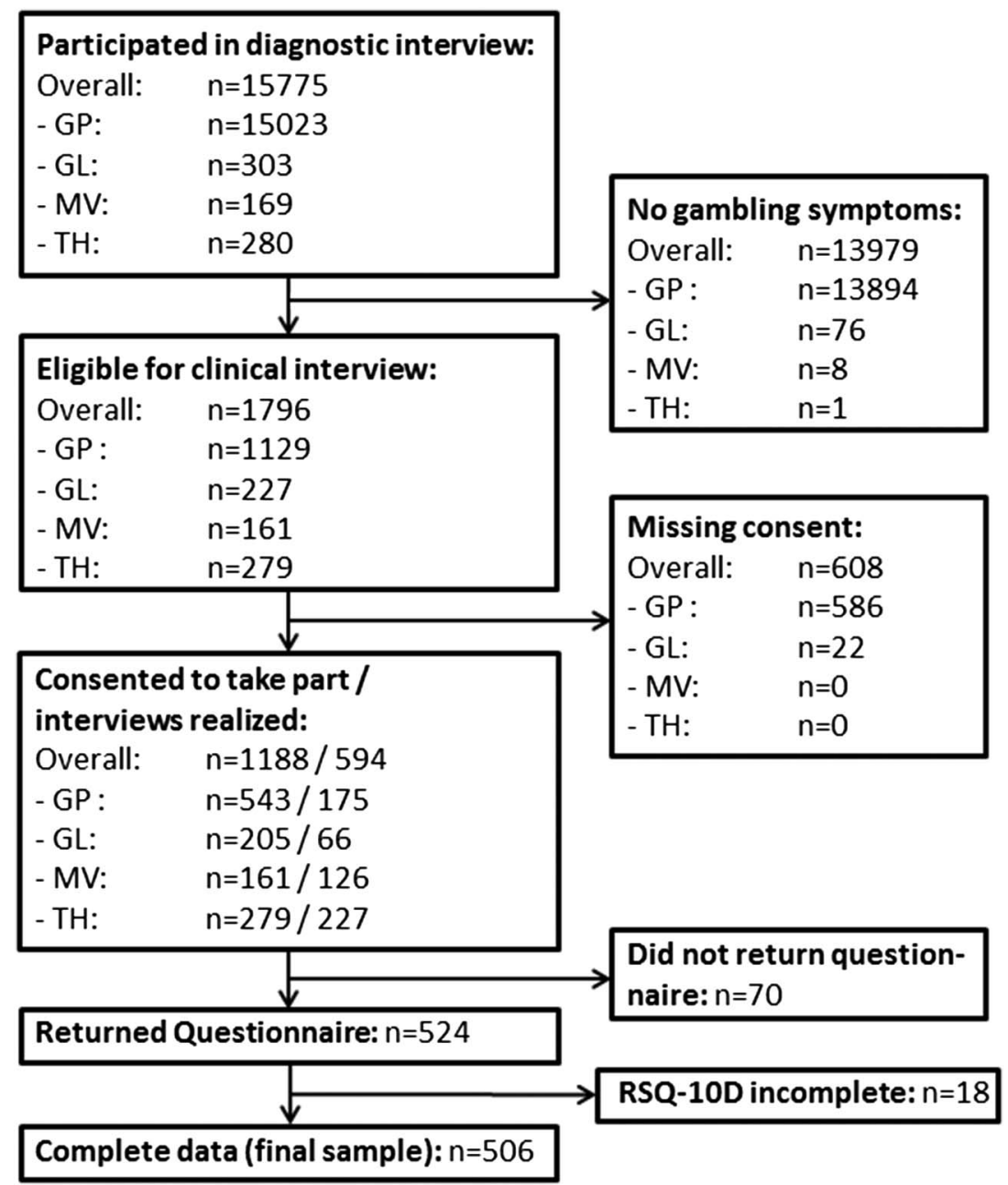

Figure 1. Participant flow. GP: participants from the general population sample; GL: participants from gambling locations; MV: media volunteers; TH: participants undergoing treatment or seeking help

Table 2. Distribution of the symptom counts for symptoms of pathological gambling and symptoms of depression

\begin{tabular}{lcccrr}
\hline $\begin{array}{l}\text { Count of } \\
\text { gambling } \\
\text { symptoms }\end{array}$ & $n$ & \multicolumn{5}{c}{$\begin{array}{c}\text { Count of } \\
\text { depressive } \\
\text { symptoms }\end{array}$} & \multicolumn{1}{c}{$n$} & \multicolumn{1}{c}{$\%$} \\
\hline 1 & 41 & 8.10 & 0 & 154 & 30.43 \\
2 & 29 & 5.73 & 1 & 7 & 1.38 \\
3 & 32 & 6.32 & 2 & 15 & 2.96 \\
4 & 29 & 5.73 & 3 & 29 & 5.73 \\
5 & 31 & 6.13 & 4 & 38 & 7.51 \\
6 & 29 & 5.73 & 5 & 41 & 8.10 \\
7 & 51 & 10.08 & 6 & 57 & 11.26 \\
8 & 58 & 11.46 & 7 & 65 & 12.85 \\
9 & 113 & 22.33 & 8 & 58 & 11.46 \\
10 & 93 & 18.38 & 9 & 42 & 8.30 \\
Total & 506 & 100 & - & 506 & 100 \\
\hline & & & & &
\end{tabular}

participants reported a mean of $4.19(S D=3.28)$ depressive symptoms. Pathological gamblers reported the highest number of depressive symptoms $(M=4.47, S D=3.22)$, followed by at-risk $(M=3.4, S D=3.37)$ and problem gamblers $(M=3.38, S D=3.32)$. The mean values significantly differed between the three groups of gamblers $(F=5.36$, $p=.005)$. Single comparisons revealed significant differences between pathological and at-risk gamblers as well as problem gamblers ( $p=.036$ and $p=.046$, respectively). At-risk and problem gamblers did not significantly differ $(p=1.000)$.

Ruminative brooding and reflection. The brooding and reflection scores in our sample were significantly correlated $(r=.58, p<.001)$. The mean score for $\mathrm{RB}$ was $11.69(S D=$ 3.30, range: 5-20). Pathological gamblers reported the highest brooding scores $(M=12.06, S D=3.32)$, followed by problem $(M=11.38, S D=3.04)$ and at-risk gamblers $(M=10, S D=2.91)$. The mean values significantly differed between the three groups of gamblers $(F=12.34, p<.001)$. Single comparisons revealed significant differences between at-risk and problem gamblers as well as pathological gamblers ( $p=.046$ and $p<.001$, respectively). Between problem and pathological gamblers, no significant differences were observed $(p=.377)$.

The mean for RR was $10.46(S D=3.03$, range: $5-20)$. Pathological gamblers reported the highest reflection scores $(M=10.65, S D=2.96)$, followed by problem $(M=10.23$, $S D=2.89)$ and at-risk gamblers $(M=9.7, S D=3.37)$. The mean values significantly differed between the three groups 
of gamblers $(F=3.11, p=.045)$. Single comparisons revealed significant differences only between at-risk and pathological gamblers $(p=.049)$ but not between at-risk and problem gamblers $(p=.949)$ or problem and pathological gamblers $(p=.954)$.

\section{Uni- and multivariate median regression analyses}

Univariate median regression analyses. In the univariate median regression analyses, RB but not RR was significantly associated with SPG. The association was positive, meaning that at the median, an increase in RB was associated with an increase in the severity of gambling problems. The absolute number of depressive symptoms, low education level, being unmarried, male sex, and being reactively recruited also showed significant positive associations with SPG at the median. Being employed showed a significant negative association with SPG, meaning that at the median, being employed was associated with less SPG. Age and having a migration background showed no significant associations with SPG at the median (c.f. Table 3).

Multivariate median regression analysis. In the multivariate median regression, RB remained significantly associated with SPG when controlling for RR, depressive symptoms, employment status, education, marital status, age, sex, and recruitment strategy. The association was positive, meaning that an increase in RB was associated with an increase in the severity of gambling problems. The data revealed no significant association of RR with SPG at the median. As in the univariate median regression, the absolute number of depressive symptoms remained significantly positively associated with SPG after controlling for the remaining variables (c.f. Table 3).

\section{Quantile regression with quantiles related to number of symptoms of pathological gambling}

Table 4 provides detailed results of the QR analyses with quantiles related to the number of SPG. When controlling for RR, depressive symptoms, sociodemographic variables, and recruitment strategy, RB was significantly associated with SPG at all quantiles except the lowest quantile related to one SPG. The association was positive for all of these quantiles, meaning that an increase in RB was associated with an increase in the severity of gambling problems at almost every severity level of problem gambling. At the quantile related to one SPG, the association was also positive but failed to reach statistical significance $(p=.079)$.

For RR, there was a statistically significant association with SPG only at the quantile related to six SPG when including $\mathrm{RB}$, depressive symptoms, sociodemographic variables, and recruitment strategy into the model. This association was negative, meaning that an increase in RR is associated with a decrease in the severity of gambling problems at this quantile (c.f. Figure 2).

When controlling for RB, RR, sociodemographic variables, and recruitment strategy, the absolute number of depressive symptoms was significantly associated with SPG at the quantiles related to four and seven or more SPG. All of the significant associations were positive. At lower levels of problem gambling severity, no significant associations between the absolute number of depressive symptoms and SPG were found when controlling for covariates.

\section{DISCUSSION}

This study aimed at analyzing the relationship between the rumination subfactors brooding and reflection and SPG. The main findings of this analysis are that (a) RB and SPG are associated and that (b) especially at subclinical levels of gambling problems, RB seems to have a larger impact on gambling behavior than depressive symptoms.

In our sample, problem and pathological gamblers reported significantly higher RB scores compared to at-risk gamblers. Univariate median regression analysis showed a significant association between RB and SPG that, in a multivariate model, could not be fully explained by depressive symptoms or other covariates. When looking at quantiles related to the number of SPG, RB was independently positively associated with SPG at all but the lowest severity level of problem gambling. Our findings suggest that RB

Table 3. Results of the univariate and multivariate median (quantile) regression analyses with symptoms of pathological gambling as the dependent measure

\begin{tabular}{|c|c|c|c|c|c|c|}
\hline \multirow[b]{2}{*}{ Independent measures } & \multicolumn{3}{|c|}{ Univariate median regressions } & \multicolumn{3}{|c|}{ Multivariate median regression } \\
\hline & Coef. & $S E$ & $p$ & Coef. & $S E$ & $p$ \\
\hline Brooding & 0.286 & 0.05 & $<.001$ & 0.177 & 0.06 & .005 \\
\hline Reflection & 0.143 & 0.08 & .073 & -0.062 & 0.07 & .347 \\
\hline Depressive symptoms & 0.200 & 0.06 & .001 & 0.121 & 0.05 & .019 \\
\hline Employed & -1.000 & 0.38 & .008 & -0.366 & 0.35 & .290 \\
\hline Lower education $^{\mathrm{a}}$ & 2.000 & 0.52 & $<.001$ & 0.764 & 0.35 & .028 \\
\hline Not married ${ }^{\mathrm{b}}$ & 1.000 & 0.47 & .031 & -0.070 & 0.37 & .850 \\
\hline Migration background & 1.000 & 0.57 & .077 & -0.108 & 0.37 & .772 \\
\hline Male & 2.000 & 0.46 & $<.001$ & 0.923 & 0.41 & .025 \\
\hline Age & 0.031 & 0.02 & .134 & -0.004 & 0.01 & .764 \\
\hline Reactive recruitment & 5.000 & 0.26 & $<.001$ & 4.254 & 0.33 & $<.001$ \\
\hline
\end{tabular}

Note. Coef:: coefficient; $S E$ : standard error.

${ }^{\mathrm{a}} \leq 10$ school years; ${ }^{\mathrm{b}}$ single, separated, divorced, and widowed. 

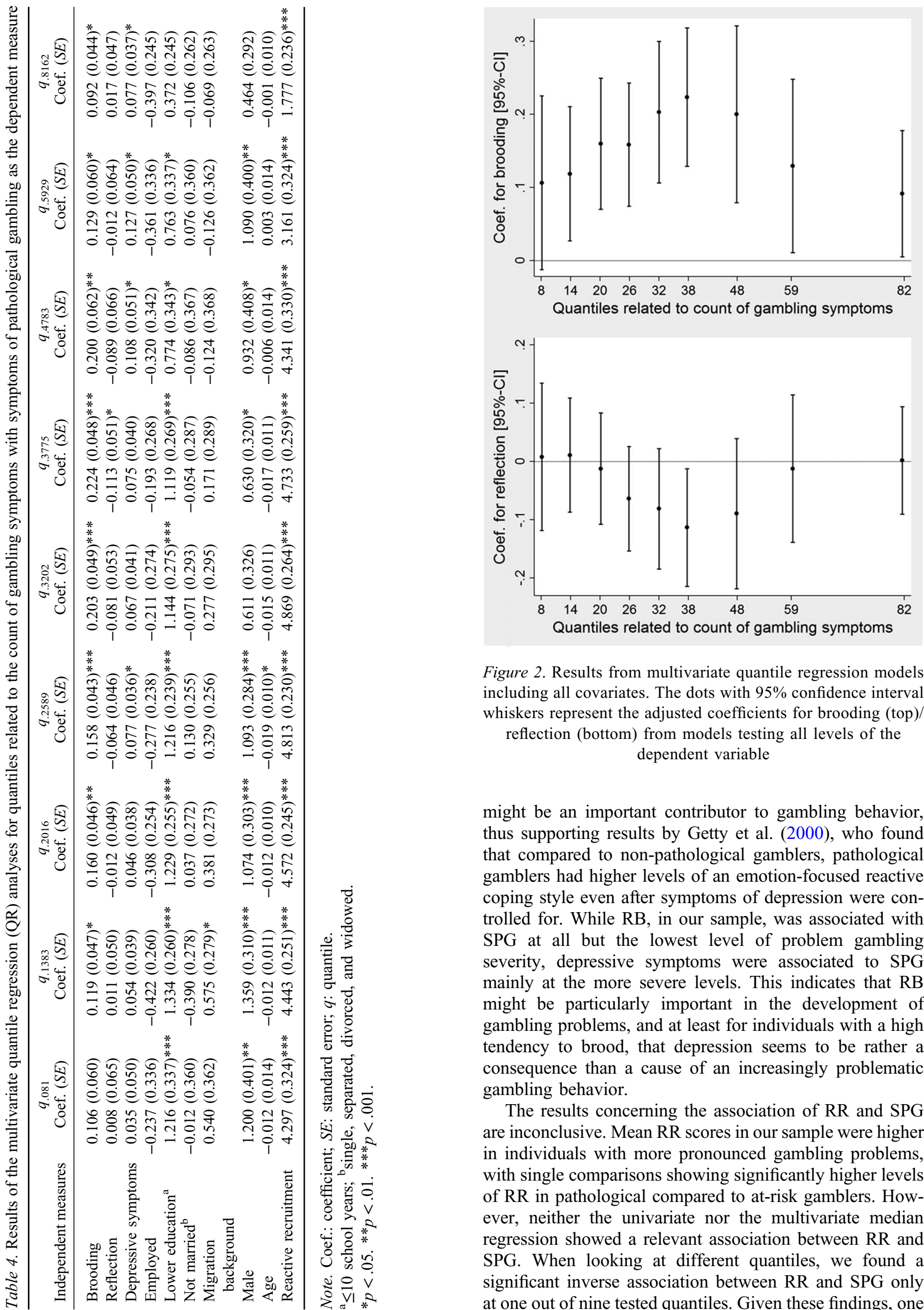

Figure 2. Results from multivariate quantile regression models including all covariates. The dots with $95 \%$ confidence interval whiskers represent the adjusted coefficients for brooding (top)/ reflection (bottom) from models testing all levels of the dependent variable

might be an important contributor to gambling behavior, thus supporting results by Getty et al. (2000), who found that compared to non-pathological gamblers, pathological gamblers had higher levels of an emotion-focused reactive coping style even after symptoms of depression were controlled for. While RB, in our sample, was associated with SPG at all but the lowest level of problem gambling severity, depressive symptoms were associated to SPG mainly at the more severe levels. This indicates that RB might be particularly important in the development of gambling problems, and at least for individuals with a high tendency to brood, that depression seems to be rather a consequence than a cause of an increasingly problematic gambling behavior.

The results concerning the association of RR and SPG are inconclusive. Mean RR scores in our sample were higher in individuals with more pronounced gambling problems, with single comparisons showing significantly higher levels of RR in pathological compared to at-risk gamblers. However, neither the univariate nor the multivariate median regression showed a relevant association between $R R$ and SPG. When looking at different quantiles, we found a significant inverse association between RR and SPG only at one out of nine tested quantiles. Given these findings, one 
might speculate that at this medium level of gambling problems, RR might have a protective effect on gambling behavior, maybe through an effective problem solving it supposedly provokes (Treynor et al., 2003). However, this part of our analysis was explorative as we did not adjust for multiple testing. Hence, these findings may be rather seen as an indication of no substantial independent association of SPG and RR, which would support the results reported by Getty et al. (2000). In conclusion, available data rather suggests that RR is of no special interest in SPG, but more research is needed to further clarify its role.

Several limitations have to be considered for this analysis (c.f. Meyer et al., 2015). First, the association between RB and SPG found in this analysis could be better explained by other factors that were not taken into account in our analysis. Second, as the data collection primarily relied on self-reports, a reporting bias cannot be ruled out. Third, a substantial part of the subjects who were called in the telephone survey refused to participate, probably leading to a selection bias in the general population sample. Fourth, we did not realize a random sample of gambling location attenders and inpatients, and the reactively recruited participants were per se selfselected. Thus, although our analyses were statistically controlled for recruitment strategy, generalization of findings to the population level might not be valid. Finally, causal interpretation of the associations found in this study is precluded by the cross-sectional nature of our data.

\section{CONCLUSIONS}

This study revealed that RB is associated with SPG and that it might be particularly important in the development of gambling problems. Furthermore, with its relations to depression as well as to gambling behavior, RB seems to be a crucial factor when it comes to explaining the high comorbidity between depressive disorders and gambling disorders (Kessler et al., 2008; Petry et al., 2005; Rizeanu, 2013). Diagnoses and treatment of ruminative tendencies should be an inherent part in the treatment of gambling problems. Depressive rumination should further be considered in the prevention of gambling disorders. However, future research based on longitudinal data is necessary to gain more insights into the underlying causal mechanisms connecting gambling problems, rumination, and depression.

Funding sources: Data collection was funded by the 16 German federal states as part of the Pathological Gambling and Epidemiology (PAGE) program. Data analysis and conducting of this study was supported by the research consortium "Addiction: Early Recognition and Intervention Across the Lifespan (AERIAL)," funded by the German Federal Ministry of Education and Research (grant no. FKZ 01EE1406F). The authors would like to acknowledge the support of the Article Processing Charge from the DFG (German Research Foundation, 393148499) and the Open Access Publication Fund of the University of Greifswald. The funding organizations had no further role in the collection, analysis, and interpretation of data.
Authors' contribution: UJ, CM, and H-JR outlined the concept of the underlying PAGE study in the grant proposal. $\mathrm{AB}, \mathrm{H}-\mathrm{JR}$, and $\mathrm{CM}$ were responsible for organizing the fieldwork. AB, H-JR, CM, DG, SL, and KK were responsible for preparation and checks of the data. KK, DG, SL, and $\mathrm{CM}$ took part in the data analysis and all authors contributed to the interpretation of the results. KK drafted the initial version of the manuscript and all authors revised the manuscript for important intellectual content. All authors read and approved the final manuscript.

Conflict of interest: The authors declare no conflict of interest.

\section{REFERENCES}

Afifi, T. O., Nicholson, R., Martins, S. S., \& Sareen, J. (2016). A longitudinal study of the temporal relation between problem gambling and mental and substance use disorders among young adults. Canadian Journal of Psychiatry-Revue Canadienne De Psychiatrie, 61(2), 102-111. doi:10.1177/ 0706743715625950

Cameron, A. C., \& Trivedi, P. K. (2009). Microeconometrics using stata (Vol. 5). College Station, TX: Stata Press.

Chinneck, A., Mackinnon, S. P., \& Stewart, S. H. (2016). Investigating possible reciprocal relations between depressive and problem gambling symptoms in emerging adults. Canadian Journal of Psychiatry-Revue Canadienne De Psychiatrie, 61(2), 93-101. doi:10.1177/0706743715625934

Dickerson, M. G., Baron, E., Hong, S.-M., \& Cottrell, D. (1996). Estimating the extent and degree of gambling related problems in the Australian population: A national survey. Journal of Gambling Studies, 12(2), 161-178. doi:10.1007/BF01539172

Getty, H. A., Watson, J., \& Frisch, G. R. (2000). A comparison of depression and styles of coping in male and female GA members and controls. Journal of Gambling Studies, 16(4), 377-391. doi:10.1023/A:1009480106531

Griffiths, M. (1995). The role of subjective mood states in the maintenance of fruit machine gambling behaviour. Journal of Gambling Studies, 11(2), 123-135. doi:10.1007/BF02107111

Heppner, P. P., Cook, S. W., Wright, D. M., \& Johnson, W. C. (1995). Progress in resolving problems - A problem-focused style of coping. Journal of Counseling Psychology, 42(3), 279-293. doi:10.1037/0022-0167.42.3.279

Huffziger, S., \& Kühner, C. (2012). Die Ruminationsfacetten Brooding und Reflection [The rumination components brooding and reflection. Factor structure and psychometric properties of the German version RSQ-10D]. Zeitschrift für Klinische Psychologie und Psychotherapie, 41(1), 38-46. doi:10.1026/ 1616-3443/a000118

Kessler, R. C., Hwang, I., LaBrie, R., Petukhova, M., Sampson, N. A., Winters, K. C., \& Shaffer, H. J. (2008). DSM-IV pathological gambling in the National Comorbidity Survey Replication. Psychological Medicine, 38, 1351-1360. doi:10.1017/s0033291708002900

Koenker, R. (2005). Quantile regression. Cambridge, UK: Cambridge University Press.

McCormick, R. A., Russo, A. M., Ramirez, L. F., \& Taber, J. I. (1984). Affective disorders among pathological gamblers 
seeking treatment. The American Journal of Psychiatry, 141(2), 215-218. doi:10.1176/ajp.141.2.215

Meyer, C., Bischof, A., Westram, A., Jeske, C., de Brito, S., Glorius, S., Schön, D., Porz, S., Gürtler, D., Kastirke, N., Hayer, T., Jacobi, F., Lucht, M., Premper, V., Gilberg, R., Hess, D., Bischof, G., John, U., \& Rumpf, H. J. (2015). The "Pathological Gambling and Epidemiology" (PAGE) study program: Design and fieldwork. International Journal of Methods in Psychiatric Research, 24(1), 11-31. doi:10.1002/mpr.1458

Nolen-Hoeksema, S. (1991). Responses to depression and their effects on the duration of depressive episodes. Journal of Abnormal Psychology, 100(4), 569-582. doi:10.1037/0021-843X.100.4.569

Nolen-Hoeksema, S., Wisco, B. E., \& Lyubomirsky, S. (2008). Rethinking rumination. Perspectives on Psychological Science, 3(5), 400-424. doi:10.1111/j.1745-6924.2008.00088.x

Petry, N. M., Stinson, F. S., \& Grant, B. F. (2005). Comorbidity of DSM-IV pathological gambling and other psychiatric disorders: Results from the national epidemiologic survey on alcohol and related conditions. Journal of Clinical Psychiatry, 66(5), 564-574. doi:10.4088/JCP.v66n0504
Rizeanu, S. (2013). Pathological gambling and depression. In C. Vasile, M. Anitei, \& M. Chraif (Eds.), Psiworld 2012 (Vol. 78, pp. 501-505). Amsterdam, The Netherlands: Elsevier Science Bv.

StataCorp. (2015). Stata Statistical Software: Release 14. College Station, TX: StataCorp LP.

Treynor, W., Gonzalez, R., \& Nolen-Hoeksema, S. (2003). Rumination reconsidered: A psychometric analysis. Cognitive Therapy and Research, 27(3), 247-259. doi:10.1023/A: 1023910315561

Wittchen, H., Beloch, E., Garczynski, E., Holly, A., Lachner, G., Perkonigg, A., Vodermaier, A., Vossen, A., Wunderlich, U., \& Zieglgänsberger, S. (1995). Münchener Composite International Diagnostic Interview (M-CIDI). München, Germany: Max-Planck-Institut für Psychiatrie.

World Health Organization. (2009). The World Mental Health Survey Initiative. Computer Assisted Personal Interview (CAPI V21.1.1). Gambling section. Retrieved December 31, 2013, from http://www.hcp.med.harvard.edu/wmhcidi/instruments_ capi.php 